\title{
Efficacy of nano-carbonate apatite dentifrice in relief from dentine hypersensitivity following non-surgical periodontal therapy: a randomized controlled trial
}

\author{
Pei-Hui Ding ${ }^{1,2+}$, Anna Dai ${ }^{2,3+}$, Hua-Jiao Hü ${ }^{2,3}$, Jia-Ping Huang ${ }^{2,3}$, Jia-Mei Liu ${ }^{2,3}$ and Li-Li Chen ${ }^{2,3^{*}}$ (D)
}

\begin{abstract}
Background: Dentine hypersensitivity $(\mathrm{DH})$ could occur or intensify after non-surgical periodontal therapy because of the exposure of dentine tubules, but currently no gold standard exists to treat $\mathrm{DH}$. It has been demonstrated that nano-sized particles presented potential for dentine tubules blocking and remineralization. This randomized controlled trial aimed to investigate the efficacy of dentifrice containing nano-carbonate apatite (n-CAP) in reducing dentine hypersensitivity $(\mathrm{DH})$ after non-surgical periodontal therapy.
\end{abstract}

Methods: 48 periodontitis patients with DH were included in this clinical trial. After non-surgical periodontal therapy, patients included were randomized to test and control group and the respective dentifrices were applied at chairside, after which they were instructed to brush teeth with the allocated dentifrices twice a day at home. Periodontal parameters were recorded at baseline and the last follow-up. DH was measured by air-blast test and recorded by visual analogue scale (VAS) and Schiff sensitivity scale at baseline, after polishing (0 week) and 2/4/6 weeks.

Results: 45 participants completed the follow-up. Periodontal parameters were improved and comparable between groups. Significant reduction in DH was observed in both groups at all time-points compared to baseline in terms of VAS and Schiff score. The test group achieved significantly greater relief from hypersensitivity compared with the control group after 4-week at-home use (for change of VAS, test group: $2.27 \pm 2.47$ versus control group: $1.68 \pm 2.24$, $p=0.036$; for change of Schiff, test group: $0.94 \pm 0.92$ versus control group: $0.61 \pm 0.83, p<0.001)$. The 6 -week results showed borderline significance between groups in terms of change of Schiff $(p=0.027)$ and no significance in terms of change of VAS $(p=0.256)$.

(Continued on next page)

\footnotetext{
* Correspondence: chenlili_1030@zju.edu.cn

${ }^{\dagger}$ Pei-Hui Ding and Anna Dai contributed equally to this work.

${ }^{2}$ Key Laboratory of Oral Biomedical Research of Zhejiang Province, Zhejiang University School of Stomatology, Hangzhou, China

${ }^{3}$ Department of Periodontology, the Second Affiliated Hospital of Zhejiang University School of Medicine, No. 88 Jiefang Road, Hangzhou 310009, China Full list of author information is available at the end of the article
}

C C The Author(s). 2020 Open Access This article is licensed under a Creative Commons Attribution 4.0 International License, which permits use, sharing, adaptation, distribution and reproduction in any medium or format, as long as you give appropriate credit to the original author(s) and the source, provide a link to the Creative Commons licence, and indicate if changes were made. The images or other third party material in this article are included in the article's Creative Commons licence, unless indicated otherwise in a credit line to the material. If material is not included in the article's Creative Commons licence and your intended use is not permitted by statutory regulation or exceeds the permitted use, you will need to obtain permission directly from the copyright holder. To view a copy of this licence, visit http://creativecommons.org/licenses/by/4.0/. The Creative Commons Public Domain Dedication waiver (http://creativecommons.org/publicdomain/zero/1.0/) applies to the data made available in this article, unless otherwise stated in a credit line to the data. 
(Continued from previous page)

Conclusions: Home-use of n-CAP based dentifrice had some benefit on alleviation of DH following non-surgical periodontal therapy after 4 weeks compared to the control product.

Trial registration: Chinese Clinical Trials Registry (No. ChiCTR-IPR-17011678, http://www.chictr.org.cn/, registered 16 June, 2017).

Keywords: Dentin hypersensitivity, Dentifrices, Randomized controlled trial, Periodontitis

\section{Background}

Dentine hypersensitivity $(\mathrm{DH})$ is characterized by an acute pain arising from exposed dentine in response to external stimuli, including thermal, evaporative, tactile, osmotic or chemical stimuli, which cannot be ascribed to other forms of dental defect or disease [1, 2]. The most widely accepted hypothesis to explain the mechanism of DH is Brannstrom's hydrodynamic theory [3]. According to this theory, exposed tubules on dentine surface allow the rapid movement of dentinal fluid, indirectly stimulating the pulp nerve terminals and consequently causing sharp and shooting pain. Further histological study revealed that tubule diameters were significantly larger in hypersensitive area compared to non-sensitive surface which is usually covered by a smear layer [4].

DH can arise as a result of enamel loss caused by erosion, abrasion, attrition, as well as cementum loss typically subsequent to gingival recession [5]. Dental professionals may also contribute to cementum removal and tubule exposure by root surface instrumentations [6]. It is common that periodontitis patients complain about increased sensitivity following scaling and root planing [7]. The prevalence of $\mathrm{DH}$ in the published literature varies from 62.5 to $90 \% 1$ day after non-surgical periodontal therapy [7]. In view of the high prevalence, a prophylaxis method to desensitize $\mathrm{DH}$ after root debridement procedure would be helpful for patients.

Over years numerous regiments have been recommended for the relief of $\mathrm{DH}$, ranging from home-use desensitizing dentifrices and mouthwashes to in-office application products such as varnishes, dentine-bonding agents, composite resins, glass ionomer cements and laser [5]. Owing to low cost, easy use, home application and daily habit, desensitizing dentifrices could be considered as preferable agents in the routine management of DH. The Canadian Advisory Board on Dentine Hypersensitivity [2] suggested that home-care approach was the first choice to treat DH, such as desensitizing toothpaste. If the symptom was not alleviated, an in-office therapy would then be recommended.

Active ingredients like strontium [8] and potassium salts $[9,10]$ were widely used to act on the pulp nerve mechanoreceptors and block painful stimuli. However, $\mathrm{DH}$ might reoccur as the concentration of these ions decreased. The results in vitro demonstrated that several substances such as calcium chloride [11], fluoride [12], bioactive glass-ceramic [13] promoted the formation of a superficial pellicle over dentinal tubules, but this did not inevitably happen because the small particles could be dissolved or washed from the tubules in the in vivo environment after day-to-day activity [5]. Some studies have revealed that nano-sized particles would adhere to the dentine surface increasingly because of high surface energy, indicating their potential for occluding tubules $[14,15]$. More importantly, the remineralization potential of nano-sized particles enabled them to maintain continuous capacity for exposed dentine surface repair $[14,16,17]$. A recent meta-analysis showed that nanohydroxyapatite $\left[\mathrm{Ca}_{10}\left(\mathrm{PO}_{4}\right)_{6}(\mathrm{OH})_{2}\right]$ provided greater $\mathrm{DH}$ relief when compared to placebo or negative control [18]. Carbonate apatite [CAP, $\left.\mathrm{Ca}_{10}\left(\mathrm{PO}_{4} \cdot \mathrm{CO}_{3}\right)_{6}(\mathrm{OH})_{2}\right]$, whose structure was modified from hydroxyapatite, is chemically similar to the main inorganic component of dentine [19]. A new dentifrice containing 20\% nanosized CAP (n-CAP) has shown to occlude dentinal tubules of $77.4 \%$ more than that of the control group in vitro, which indicated a potential use in $\mathrm{DH}$ [15]. A recent clinical trial initially reported the desensitizing effect of this dentifrice in 2 weeks and the effect could be maintained for the later 2 weeks [20].

However, no published study has yet evaluated the effect of $\mathrm{n}$-CAP in treating DH that occurred after scaling and root planing therapy. The present double-blind randomized controlled trial aimed to investigate the efficacy of the dentifrice containing 20\% n-CAP in reducing DH following non-surgical periodontal therapy after immediate in-office application and during the 6-week homeuse period.

\section{Methods}

This randomized, controlled, parallel-group clinical trial was performed from June 2017 to July 2018 in the Department of Periodontology of the Second Affiliated Hospital of Zhejiang University School of Medicine, Hangzhou (SAHZU), China. This randomized trial followed the CONSORT 2010 Checklist protocol [21]. The study protocol was approved by the Human Research Ethics Committee of SAHZU (NO. 2017037) and registered at the Chinese Clinical Trials Registry (No. 
ChiCTR-IPR-17011678, http://www.chictr.org.cn/) before patient enrolment and conducted in full accordance with the Declaration of Helsinki.

\section{Inclusion and exclusion criteria}

Each participant was fully informed about the research and signed the written consent prior to enrollment. The patient inclusion criteria were: (1) aged 18 to 60 years; (2) good general health without systemic disease; (3) no smoking history or quitting smoking for at least 2 years; (4) with a diagnosis of moderate or severe periodontitis $[22,23]$, who needed to be treated by non-surgical periodontal treatment (scaling and root planing) in one session. Briefly, moderate periodontitis was diagnosed as clinical attachment level (CAL) $=3$ or $4 \mathrm{~mm}$ and probing depth (PD) $\geq 4$ in $\geq 2$ non-adjacent teeth, and severe periodontitis was diagnosed as $\mathrm{CAL} \geq 5$ and $\mathrm{PD} \geq 5$ in $\geq 2$ non-adjacent teeth; (5) with at least three teeth in the buccal face existing dentine hypersensitivity [visual analogue scale $(\mathrm{VAS}) \geq 2.0$ ] after non-surgical periodontal therapy, which was evaluated by air-blast test. The patient exclusion criteria were: (1) patients with gross oral mucosal disease (oral lichen planus, oral ulcer, et al), reflux or bulimia, extremely advanced periodontitis who cannot endure one session non-surgical periodontal therapy; (2) patients receiving surgical or nonsurgical periodontal therapy within 12 months; (3) patients using desensitizing agents in the past 6 months; (4) hypersensitive teeth with mobility greater than $1^{\circ}$; (5) hypersensitive teeth which were the second or third molars; (6) hypersensitive teeth with extensive and/or defective restorations, suspected caries, pulpitis or cracked enamel; (7) patients with chronic use of antihistamines, anticonvulsants, sedatives, antidepressants, tranquilizers or daily analgesics within 1 month; (8) pregnant or lactating females; (9) patients presenting allergies to the test product, or ever allergic to oral care consumer products; (9) patients who have participated in another desensitizing dentifrice study.

\section{Sample size estimation}

The main outcome was the VAS difference across groups between the mean changes in air-blast test from baseline evaluation to the end of the follow-up. According to previous data $[24,25]$, the expected baseline mean VAS score was $5.5 \pm 2.0$. The hypothesized mean VAS score at the end of the follow-up was $2.0 \pm 1.9$ for the test dentifrice and $3.5 \pm 1.8$ for the control product, and the mean changes were $3.5 \pm 1.8$ and $2.0 \pm 1.5$, respectively. Using an unpaired $t$-test and assuming an $\alpha$ error $=0.05$, power $=80 \%$ (two-tailed comparison), a minimum of 21 participants per group were requested using a single allocation ratio (1:1) (G*Power version 3.1 for Mac, Franz Haul, University of Kiel, Germany). To compensate for the possibility of $15 \%$ dropouts, 48 patients per group were aimed to be recruited.

\section{Non-surgical periodontal therapy}

The enrolled patients received full-mouth scaling and root planing in one session. The non-surgical periodontal therapy was performed using ultrasonic scaler (P5 Newtron, Acteon Satelec, France) combined with hand instruments (Gracey curettes, Hu-Friedy, Chicago, USA) after local anesthesia by $4 \%$ atticacaine and $1 / 100,000$ adrenaline (Primacaine, Produits Dentaires Pierre Rolland, France). After the therapy, chairside irrigation with 0.12\% Chlorhexidine (Koutai, South China Pharmaceutical, China) for $1 \mathrm{~min}$. All patients were taught to brush teeth by modified bass technique using the same kind of soft toothbrush (Systema, Lion, Japan) provided, twice a day for 3 min during the 6-week trial.

All periodontal treatments were performed by the same experienced periodontist PHD, who was masked from the patient allocation during the whole study.

\section{Clinical evaluation}

Baseline periodontal parameters were recorded before non-surgical periodontal therapy. Periodontal examination was performed for each tooth including gingival recession (GR), probing depth (PD), bleeding on probing (BOP) and clinical attachment level (CAL).

Baseline air-blast evaluation (post-scaling evaluation) for each tooth was taken $12-24 \mathrm{~h}$ after scaling and root planing. First, the tooth was isolated by the cotton roll from the adjacent teeth. Next, a blast of air from a standard dental unit syringe at $60 \pm 5 \mathrm{psi}$ at $18-22^{\circ} \mathrm{C}$ was directed onto the exposed middle $1 / 3$ buccal surface for $1 \mathrm{~s}$ at a distance of approximately $10 \mathrm{~mm}$. Then, each patient reported the sensitivity he/she sensed using the Visual Analogue Scale (VAS) [26] and Schiff Cold Air Sensitivity scale [27]. VAS scale was scoring from 0 (no pain) to 10 (intense pain). Schiff scale was scored from 0 to 3: score " 0 " means no response, score " 1 " means response without request of discontinuation of stimulus, score "2" means response with request of discontinuation of stimulus, and score "3" means pain with request of discontinuation of stimuli.

According to the baseline evaluation, patients who presented at least three hypersensitive teeth with baseline VAS $\geq 2.0$ were suitable to be included. 48 patients were sequentially enrolled by the dentist $\mathrm{AD}$ and randomly assigned to either test or control group.

Once enrolled, each patient would immediately receive one consecutive 5-s polishing of the assigned dentifrices by rubber cup at a moderate speed (about $1000 \mathrm{rpm}$ ) to all teeth by the same dentist JPH. The dentifrices were as follows: (1) test group: n-CAP dentifrice containing 20\% n-CAP (Dentiguard Sensitive, Daewoong Co, 
Korea); (2) control group: calcium carbonate-based dentifrice free of $n$-CAP or other desensitizing ingredients (Honghua, Saky, China). Both of the dentifrices were without any form of fluoride. The two dentifrices had identical appearance, which were over-wrapped to hide their original packages and labeled with different numbers from 1 to 48 . Neither investigators nor patients knew the codes. After application, each patient was subjected to post-polishing evaluation (0-week evaluation) of DH. Thereafter, patients would be called back to evaluate $\mathrm{DH}$ after 2,4 and 6 weeks. At the 6-week follow-up, the same periodontal examination as baseline was repeated. Participants were instructed to use only the assigned products to brush teeth twice a day throughout the 6-week trial. They were also instructed not to eat acidic food before toothbrushing and $1 \mathrm{~h}$ before the $\mathrm{DH}$ evaluation. Participant adherence to dentine hypersensitivity treatment was evaluated by inquiry and examination of the remaining volume of dentifrice at each follow-up.

All periodontal and hypersensitivity outcomes were measured by the same experienced examiner $\mathrm{HJH}$, who had been well-trained before patient enrolment. The same procedure as employed at baseline was used as the standardized method throughout the clinical trial. At each visit, the occurrence of potential adverse effects was assessed by investigators by both intraoral examination and patient inquiry.

\section{Randomization and allocation concealment}

Simple randomization was adopted in this study. Random allocation list was generated using a computer program (Rand function, Excel 2016 for Mac, Microsoft, Redmond, VA, USA) by JML before patient recruitment. The assigned dentifrices were saved in opaque envelopes in advance. Other investigators only knew the number over the envelope but not the allocation sequence, and thus they were blind to the group allocation during the whole research period.

\section{Statistical analyses}

The data of patients who adhered to the assigned intervention and completed the predefined process were included in the statistical analysis. Teeth with baseline VAS $\geq 2$ and baseline Schiff score $\geq 1$ were included. The normality of data was assessed by Shapiro-Wilk test. Data with skewness was converted through logarithmic conversion before analysis. Intragroup and intergroup comparisons were analyzed by paired $t$ test and independent $t$ test, respectively. The results of VAS and Schiff score were analyzed using the mixed linear model adjusted to age, gender, tooth type as well as the number of teeth per participant contributed to the air-blast test. The corresponding baseline results of VAS or Schiff were covariates. All statistical analyses were performed using SPSS (version 24.0 for Mac, Chicago, IL, USA). A two-tailed $p$ value $<0.05$ was considered significant. Primary outcomes were change of VAS and change of Schiff at all evaluation stages.

\section{Results}

Forty-eight patients (24 patients in each group) were initially included in the study and 45 participants completed the 6-week follow-up finally (Fig. 1). A total of 199 and 188 teeth were evaluated in the n-CAP and control group, respectively. Among the three patients who were lost to follow-up, two were unwilling to attend the follow-up for working hour limitation and the other one was unable to return to the hospital due to a car accident. The patient age $(34.00 \pm 7.63$ versus $38.91 \pm 7.96$ years old for test and control), gender (ratio of male: 11/ 23 versus 9/22 for test and control) and number of hypersensitivity teeth for each participant were presented in Table S1.

The baseline periodontal parameters (CAL, PD, GR, BOP at patient level and cal, pd., gr at tooth level) were statistically comparable between the two groups (Table 1 ). The 6-week periodontal measurements (CAL, PD, BOP, $\mathrm{cal}, \mathrm{pd}$ ) were significantly reduced for both groups compared with the baseline measurements $(p<0.001)$. The outcomes of gingival recession were significantly increased for both groups $(p<0.001)$ and no statistical difference was shown between the groups $(p>0.05)$.

$\mathrm{DH}$ of each tooth was tested by air stimuli and recorded through VAS and Schiff scores. VAS and Schiff scores demonstrated no statistically significant difference at baseline between test and control groups $(p>0.05$; Table 2, Table 3). For the 0-, 2-, 4- and 6-week evaluation, the reduction patterns of VAS and Schiff scores showed a similar trend and a significant desensitizing result in both groups (Fig. 2).

When comparing the VAS results between groups, test group showed significantly greater relief in $\mathrm{DH}$ than control group in the 4-week evaluation $(p=0.005)$. The hypersensitivity change of VAS for those sites was also statistically significant between groups at 4-week evaluation $(p=0.036)$. However, after 6 weeks, there was no significance between groups in terms of change of VAS $(p=0.256)$. For the results of Schiff score, test group showed more reduction in DH after 4 weeks $(p<0.001)$ and 6 weeks $(p=0.027)$ and the hypersensitivity change of Schiff score showed similar results.

No adverse effects on oral tissues observed or reported by participants throughout the 6-week follow-up.

\section{Discussion}

The present clinical trial investigated the efficacy of $n$ CAP dentifrice in desensitizing hypersensitivity after 


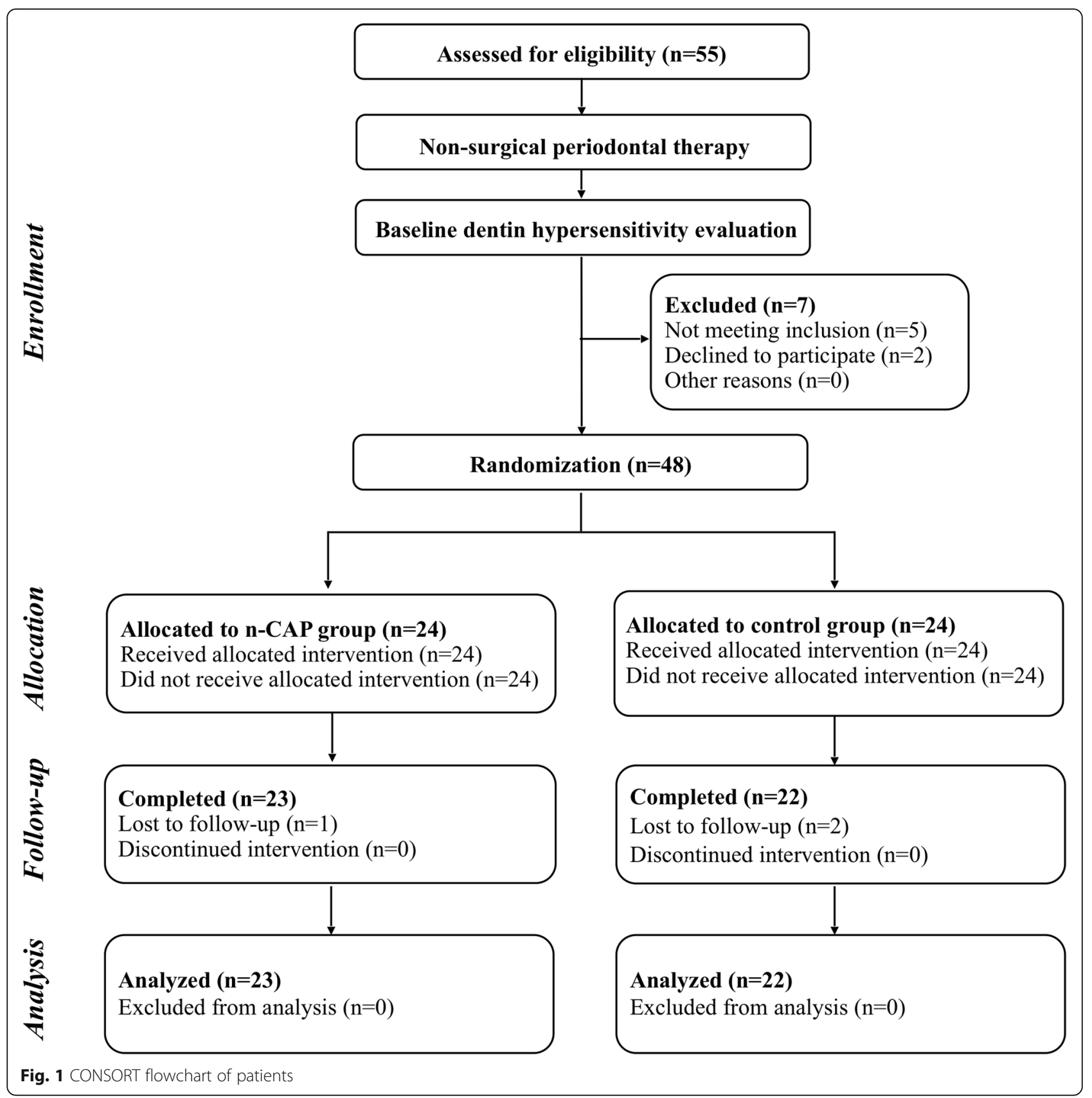

Table 1 Periodontal parameters in patient level (PD, GR, CAL, BOP) and hypersensitive tooth level (pd, gr, cal) by evaluation stage and group (mean \pm standard deviation)

\begin{tabular}{|c|c|c|c|c|c|c|c|c|}
\hline \multicolumn{9}{|c|}{ periodontal parameters } \\
\hline \multirow[t]{2}{*}{ evaluation stage } & \multirow[t]{2}{*}{ group } & \multicolumn{4}{|c|}{ patient level $(n=45)$} & \multicolumn{3}{|c|}{ hypersensitive tooth level $(n=387)$} \\
\hline & & PD $(\mathrm{mm})$ & $\mathrm{GR}(\mathrm{mm})$ & CAL (mm) & BOP (\%) & $\mathrm{pd}(\mathrm{mm})$ & $\operatorname{gr}(\mathrm{mm})$ & cal $(\mathrm{mm})$ \\
\hline \multirow[t]{2}{*}{ baseline } & test & $3.65 \pm 1.16^{\#}$ & $0.84 \pm 1.00^{\#}$ & $4.50 \pm 1.52^{\#}$ & $41.03 \pm 20.86^{\#}$ & $3.60 \pm 1.23^{\#}$ & $1.16 \pm 0.99^{\#}$ & $4.74 \pm 1.45^{\#}$ \\
\hline & control & $3.73 \pm 1.19$ & $0.90 \pm 1.02$ & $4.63 \pm 1.52$ & $35.53 \pm 13.17$ & $3.44 \pm 1.10$ & $1.23 \pm 0.95$ & $4.75 \pm 1.30$ \\
\hline \multirow[t]{2}{*}{ 6-weeks } & test & $2.68 \pm 0.71^{\#, * * *}$ & $1.06 \pm 1.05^{\#, * * *}$ & $3.74 \pm 1.36^{\#, * * * *}$ & $19.19 \pm 8.91^{\#, * * *}$ & $2.53 \pm 0.69^{\#, * * *}$ & $1.33 \pm 0.95^{\#, * * *}$ & $3.82 \pm 1.21^{\#, * * *}$ \\
\hline & control & $2.74 \pm 0.86^{* * *}$ & $1.14 \pm 1.10^{* * *}$ & $3.90 \pm 1.44^{* * *}$ & $20.73 \pm 6.45^{* * *}$ & $2.52 \pm 0.68^{* * *}$ & $1.47 \pm 1.16^{* * *}$ & $4.01 \pm 1.30^{* * *}$ \\
\hline
\end{tabular}

$P D \& p d$ pocket depth, GR \& gr gingival recession, $C A L \&$ cal clinical attachment loss, BOP bleeding on probing

\#: not statistically significantly different from the control group by independent $t$ test $(p>0.05)$

${ }^{* * *}:$ statistically significantly different from baseline by paired $t$ test $(p<0.001)$ 
Table 2 Hypersensitivity evaluation by visual analogue scale (VAS) and change of VAS scores by evaluation stage and group (mean and standard deviation)

\begin{tabular}{llll}
\hline Evaluation stage & \multicolumn{3}{l}{ Mean (SD) } \\
\cline { 2 - 4 } & test $(n=199)$ & $\operatorname{ctrl}(n=188)$ & $p$ \\
\hline post-scaling (baseline) & $4.40 \pm 1.96$ & $4.38 \pm 2.23$ & 0.820 \\
post-polishing (0 week) & $3.58 \pm 2.40^{+}$ & $3.63 \pm 2.63^{\dagger}$ & 0.800 \\
$\mathbf{2}$ weeks & $2.62 \pm 1.85^{\dagger}$ & $2.96 \pm 2.12^{\dagger}$ & 0.197 \\
$\mathbf{4}$ weeks & $2.13 \pm 1.76^{\dagger}$ & $2.71 \pm 2.17^{\dagger}$ & $0.005^{* *}$ \\
$\mathbf{6}$ weeks & $1.98 \pm 1.72^{+}$ & $2.38 \pm 2.10^{\dagger}$ & 0.098 \\
change of VAS $\mathbf{0}$ & $0.83 \pm 1.95$ & $0.76 \pm 1.64$ & 0.818 \\
change of VAS $\mathbf{2}$ & $1.77 \pm 2.11$ & $1.43 \pm 2.14$ & 0.344 \\
change of VAS $\mathbf{4}$ & $2.27 \pm 2.47$ & $1.68 \pm 2.24$ & $0.036^{*}$ \\
change of VAS $\mathbf{6}$ & $2.42 \pm 2.35$ & $2.01 \pm 2.27$ & 0.256 \\
\hline
\end{tabular}

${ }^{\dagger}$ : statistically significantly different from baseline VAS by paired $t$ test $(p<0.001)$

${ }^{*}:$ statistically significantly different from control group by mixed linear model $(p<0.05)$

${ }^{* *}$ : statistically significantly different from control group by mixed linear model $(p<0.01)$

non-surgical periodontal therapy compared with a control dentifrice in the continuous 6-week follow-up. Statistically significant decrease in DH was observed immediately after in-office application compared to baseline in both groups, while there is no difference between the test and control groups. The use of n-CAP could provide a significant reduction of DH after 4-week home-use. The 6-week comparison between groups only showed borderline significance.

Baseline and final (6 weeks) periodontal parameters (BOP, PD, GR, CAL, pd., gr, cal) and baseline air-blast

Table 3 Hypersensitivity evaluation by Schiff score and change of Schiff scores by evaluation stage and group (mean and standard deviation)

\begin{tabular}{llll}
\hline Evaluation stage & \multicolumn{3}{l}{ Mean (SD) } \\
\cline { 2 - 4 } & test $(\mathrm{n}=199)$ & $\mathrm{ctrl}(\mathrm{n}=188)$ & $p$ \\
\hline post-scaling (baseline) & $1.64 \pm 0.64$ & $1.58 \pm 0.63$ & 0.457 \\
post-polishing (0 week) & $1.28 \pm 0.85^{\dagger}$ & $1.21 \pm 0.85^{\dagger}$ & 0.574 \\
$\mathbf{2}$ weeks & $0.92 \pm 0.71^{+}$ & $1.04 \pm 0.75^{\dagger}$ & 0.200 \\
4 weeks & $0.69 \pm 0.71^{\dagger}$ & $0.97 \pm 0.77^{\dagger}$ & $0.000^{* * *}$ \\
6 weeks & $0.66 \pm 0.68^{+}$ & $0.84 \pm 0.78^{\dagger}$ & $0.047^{*}$ \\
change of Schiff $\mathbf{0}$ & $0.36 \pm 0.72$ & $0.37 \pm 0.64$ & 0.973 \\
change of Schiff $\mathbf{2}$ & $0.72 \pm 0.81$ & $0.54 \pm 0.78$ & 0.080 \\
change of Schiff $\mathbf{4}$ & $0.94 \pm 0.92$ & $0.61 \pm 0.83$ & $0.000^{* * *}$ \\
change of Schiff $\mathbf{6}$ & $0.97 \pm 0.90$ & $0.74 \pm 0.80$ & $0.027^{*}$ \\
\hline
\end{tabular}

${ }^{\dagger}$ : statistically significantly different from baseline Schiff score by paired $t$ test $(p<0.001)$

*: statistically significantly different from control group by mixed linear model $(p<0.05)$;

${ }^{* * *}:$ statistically significantly different from control group by mixed linear model $(p<0.001)$ hypersensitivity were measured. The results between test and control groups at baseline were statistically comparable. Based on the 6-week periodontal measurement, non-surgical periodontal therapy led to significant and similar improvement of periodontitis for both groups. The absence of significant difference in buccal gingival recession at baseline and 6 weeks between groups implied that the root surface exposure area was comparable between the two groups.

To explore the immediate desensitizing effect of the dentifrices, hypersensitivity was measured at postpolishing stage. The result indicated that the use of $n$ CAP did not benefit a lot to the relief of hypersensitivity immediately. Though the nano-particles are of high affinity [15], this did not enable them to adhere to the dentinal surface and seal the tubules in a few minutes, so immediate relief was not achieved as expected in this study. Douglas de Oliveira et al [28] reported that toothpaste containing calcium phosphate nanoparticles presented immediate relief effect. Notably, there was no significant difference between the test and control groups regarding evaporative and cold stimuli in the immediate assessment. Some in-office desensitizing agents have been reported to present instant relief from DH. A toothpaste with $15 \%$ of a calcium sodium phosphosilicate for a single professional application could provide a significant reduction of tactile sensitivity [29]. Another desensitizing paste containing $8 \%$ arginine and calcium carbonate led to instant relief from DH after a single inoffice application [24].

On the other hand, after 4-week consecutive home-use of the n-CAP dentifrice, with progressive deposit to the surface and remineralization open dentinal tubules, a stable mineralized layer would generate on the surface, thus minimizing the degree of DH. In the 4-week evaluation, the degree of tooth sensitivity went significantly lower in the test group than that in the control group. This is in line with the results of a recent systematic review, which showed that dentifrices containing nanohydroxyapatite had a significantly greater desensitizing effect than placebo or negative products in terms of evaporative and tactile after 4-week or 3-month followup [18]. Another dentifrice containing zinc-carbonate hydroxyapatite nanocrystals was also reported effective, which led to a significant reduction of the air-blast test score (mean percentage of reduction of $46 \%$ from baseline to 8-week evaluation) [30].

Interestingly, 6-week comparison between groups did not showed further significance than that of 4-week evaluation, raising the question whether 4-week results in the test group has decreased to a low DH level, leaving a minimal margin for further relief. As original data showed, there were as much as $93.1 \%$ patients in test group had Schiff score $\leq 1$ after 4 -week home-use. It was 

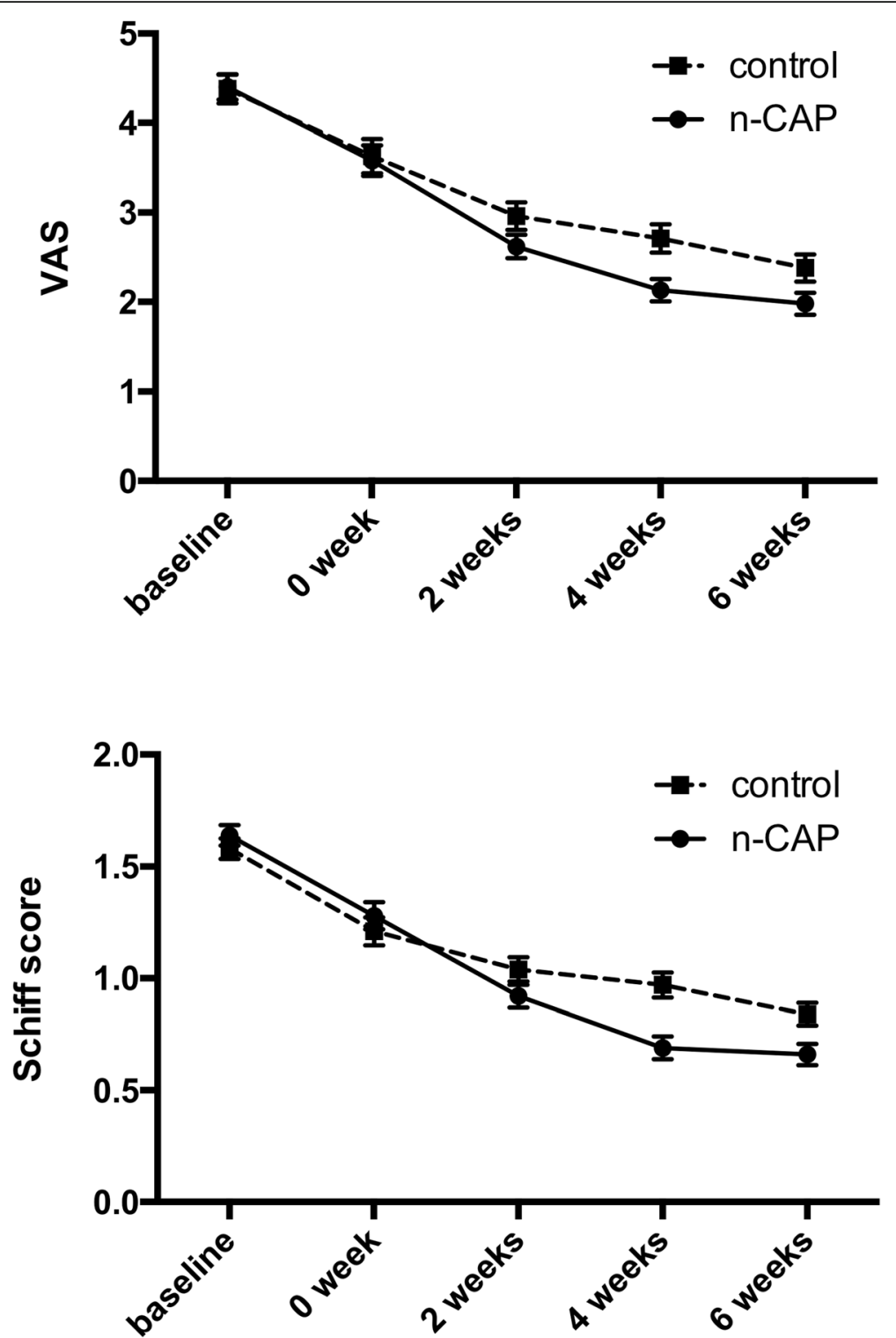

Fig. 2 Hypersensitivity evaluation by air-blast test by treatment group and evaluation stage: a mean VAS (baseline $\geq 2$ ); $\mathbf{b}$ mean Schiff score (baseline $\geq 1$ ). Data shown are mean \pm standard error

also implied in the tendency from 4-week to 6-week of Fig. 2 where a flatter line stood between these two timepoints. As a consequence, DH level went lower consistently in the control group while limited improvement occurred in the test group after 4 weeks.

It was noticed that the control group showed a similar tendency of decrease in DH during the follow-up period. It may also be attributed to the placebo effect which was common in the clinical studies of desensitizing pastes. Placebo products could reduce hypersensitivity by as much as $40 \%$ from baseline and therefore have an effect on the efficacy measurement of test dentifrice [31]. Another factor may be the Hawthorne effect. Participants tended to pay more attention to hypersensitivity and report positive outcomes in both groups. These two effects cannot be totally eliminated since the intention of the study can hardly be concealed from the participants. Our results also provided some evidence for the view that $\mathrm{DH}$ tend to self-heal over time after non-surgical periodontal therapy [32], possibly as a result of natural dentine tubule occlusion.

Air-blast test was used to test hypersensitivity in this study because it would cause more frequent pain than the tactile stimuli and involved a wider area of dentine, which indicated that the air-blast test is a sensitive and reliable method to detecting the degree of hypersensitivity [33]. The air-blast stimulus could better mimic the practical situation, since patients experienced sensitivity 
from cold water, food or air more frequently than other stimuli. In addition, many studies showed correlation between the results of air-blast test and tactile test [29, 34-36]. It is recommended that at least two independent stimuli should be applied [37], so secondary outcomes such as tactile, cold water test or subjective questionnaire could provide more supporting evidence in assessing DH degree. VAS and Schiff scores were used to exchange pain from subjective sense to objective scale. The results of these two parameters to evaluate DH were similar in the present study. Pepelassi et al. [24] also noticed that there was a strong positive correlation between VAS and Schiff scores after periodontal treatment, with the Pearson Correlation Coefficient up to 0.931 at 6 -week measurement.

One possible limitation of the present clinical trial is the lack of a positive control group. It has been recommended to set both negative and positive control groups [37], but in fact, the gold standard treatment for DH has not yet been established [38]. Pastes containing arginine [39-41], strontium acetate [30, 42] potassium ion [43, 44] or potassium nitrate [45-47] have been widely used as positive control, which were expected to facilitate rapid and considerable relief of DH. The limited sample size is another shortcoming of the present study, which may cause potential bias. Moreover, the relatively low DH level at baseline left a limited extent for improvement. Hence, clinical trials enrolling a larger number of patients with higher inclusion criteria of baseline $\mathrm{DH}$ are encouraged to confirm the present findings and determine whether this product could be recommended to the general population.

\section{Conclusions}

Within all the limitations, this randomized controlled trial showed that the application of n-CAP-based dentifrice after non-surgical periodontal therapy could had some benefit on the reduction of $\mathrm{DH}$ after 4-week athome use compared to the control dentifrice.

\section{Supplementary information}

Supplementary information accompanies this paper at https://doi.org/10. 1186/s12903-020-01157-9.

Additional file 1: Table S1. Characteristics of included patients.

\section{Abbreviations}

BOP: Bleeding on probing; CAL: Clinical attachment level;; DH: Dentine hypersensitivity; GR: Gingival recession; n-CAP: Nano-carbonate apatite; PD: Probing depth; SD: Standard deviation; VAS: Visual analogue scale

\section{Acknowledgements}

Not applicable.

\section{Authors' contributions}

PHD and $A D$ made equal contribution to designing the trial and writing the manuscript. PHD conducted the non-surgical periodontal therapy. AD enrolled patients and analyzed data. HJH was responsible for follow-up evaluation of dentine hypersensitivity and data proofreading. JPH applied the dentifrices and revised the manuscript. JML conducted the randomization. The corresponding author LLC conceived the idea, designed the study, oversaw the whole process and prepared the manuscript.

\section{Funding}

This work was supported by the National Natural Science Foundation of China (grant numbers 81771072, 81870765). PHD contributed to designing the trial, conducted the non-surgical periodontal therapy and writing the manuscript. LLC contributed to conceiving the idea, designing the study, overseeing the whole process and preparing the manuscript. The test products were provided by the company (Daewoong Co, Korea).

\section{Availability of data and materials}

The datasets used during the current study are available from the corresponding author on reasonable request.

\section{Ethics approval and consent to participate}

All procedures performed in studies involving human participants were in accordance with the ethical standards of the institutional research committee (Human Research Ethics Committee of the Second Affiliated Hospital of Zhejiang University School of Medicine, NO. 2017037) and with the 1964 Helsinki declaration and its later amendments or comparable ethical standards. Informed consent was obtained in the written version from all individual participants included in the study.

\section{Consent for publication}

Not applicable.

\section{Competing interests}

The authors declare that they have no competing interests.

\section{Author details}

'Department of Periodontology, Affiliated Hospital of Stomatology, School of Medicine, Hangzhou, China. ${ }^{2}$ Key Laboratory of Oral Biomedical Research of Zhejiang Province, Zhejiang University School of Stomatology, Hangzhou, China. ${ }^{3}$ Department of Periodontology, the Second Affiliated Hospital of Zhejiang University School of Medicine, No. 88 Jiefang Road, Hangzhou 310009, China.

Received: 25 October 2019 Accepted: 3 June 2020

Published online: 12 June 2020

\section{References}

1. Dowell P, Addy M. Dentine hypersensitivity--a review. Aetiology, symptoms and theories of pain production. J Clin Periodontol. 1983;10(4):341-50.

2. Canadian Advisory Board on Dentin H. Consensus-based recommendations for the diagnosis and management of dentin hypersensitivity. J Can Dent Assoc. 2003;69(4):221-6.

3. Brannstrom M, Astrom A. The hydrodynamics of the dentine; its possible relationship to dentinal pain. Int Dent J. 1972;22(2):219-27.

4. Absi EG, Addy M, Adams D. Dentine hypersensitivity. A study of the patency of dentinal tubules in sensitive and non-sensitive cervical dentine. J Clin Periodontol. 1987;14(5):280-4.

5. West NX. Dentine hypersensitivity: preventive and therapeutic approaches to treatment Periodontol 2000. 2008:48:31-41.

6. Wong R, Hirsch RS, Clarke NG. Endodontic effects of root planing in humans. Endod Dent Traumatol. 1989:5(4):193-6.

7. Lin YH, Gillam DG. The prevalence of root sensitivity following periodontal therapy: a systematic review. Int J Dent. 2012;2012:407023.

8. Pearce NX, Addy M, Newcombe RG. Dentine hypersensitivity: a clinical trial to compare 2 strontium densensitizing toothpastes with a conventional fluoride toothpaste. J Periodontol. 1994;65(2):113-9.

9. Orchardson R, Gillam DG. The efficacy of potassium salts as agents for treating dentin hypersensitivity. J Orofac Pain. 2000;14(1):9-19.

10. Poulsen S, Errboe M, Lescay Mevil Y, Glenny AM. Potassium containing toothpastes for dentine hypersensitivity. Cochrane Database Syst Rev. 2006; 3:CD001476. 
11. Suge T, Kawasaki A, Ishikawa K, Matsuo T, Ebisu S. Effects of pre- or postapplication of calcium chloride on occluding ability of potassium oxalate for the treatment of dentin hypersensitivity. Am J Dent. 2005;18(2):121-5.

12. Ganss C, Klimek J, Schaffer U, Spall T. Effectiveness of two fluoridation measures on erosion progression in human enamel and dentine in vitro. Caries Res. 2001;35(5):325-30.

13. Tirapelli C, Panzeri H, Lara EH, Soares RG, Peitl O, Zanotto ED. The effect of a novel crystallised bioactive glass-ceramic powder on dentine hypersensitivity: a long-term clinical study. J Oral Rehabil. 2011;38(4):253-62.

14. Ma Q, Wang T, Meng $Q, X u X, W u H, X u$ D, Chen Y. Comparison of in vitro dentinal tubule occluding efficacy of two different methods using a nanoscaled bioactive glass-containing desensitising agent. J Dent. 2017;60:63-9.

15. Lee SY, Kwon HK, Kim BI. Effect of dentinal tubule occlusion by dentifrice containing nano-carbonate apatite. J Oral Rehabil. 2008;35(11):847-53.

16. Huang S, Gao S, Cheng L, Yu H. Remineralization potential of nanohydroxyapatite on initial enamel lesions: an in vitro study. Caries Res. 2011; 45(5):460-8.

17. Tschoppe P, Zandim DL, Martus P, Kielbassa AM. Enamel and dentine remineralization by nano-hydroxyapatite toothpastes. J Dent. 2011;39(6):430-7.

18. de Melo AC, de Paula BLF, Guanipa Ortiz MI, Barauna Magno M, Martins Silva C, Cople Maia L. Clinical efficacy of nano-hydroxyapatite in dentin hypersensitivity: a systematic review and meta-analysis. J Dent. 2019;82:11-21.

19. Kim YS, Kwon HK, Kim Bl. Effect of nano-carbonate apatite to prevent restain after dental bleaching in vitro. J Dent. 2011;39(9):636-42.

20. Lee SY, Jung HI, Jung BY, Cho YS, Kwon HK, Kim Bl. Desensitizing efficacy of nano-carbonate apatite dentifrice and Er,Cr:YSGG laser: a randomized clinical trial. Photomed Laser Surg. 2015;33(1):9-14.

21. Moher D, Hopewell S, Schulz KF, Montori V, Gotzsche PC, Devereaux PJ, Elbourne D, Egger M, Altman DG. Consolidated standards of reporting trials G: CONSORT 2010 explanation and elaboration: updated guidelines for reporting parallel group randomised trials. J Clin Epidemiol. 2010;63(8):e1-37.

22. Armitage GC. Periodontal diagnoses and classification of periodontal diseases. Periodontol 2000. 2004;34:9-21.

23. Tonetti MS, Claffey N. European Workshop in Periodontology group C: Advances in the progression of periodontitis and proposal of definitions of a periodontitis case and disease progression for use in risk factor research. Group C consensus report of the 5 th European Workshop in Periodontology. J Clin Periodontol. 2005;32(Suppl 6):210-3.

24. Pepelassi E, Rahiotis C, Peponi E, Kakaboura A, Vrotsos I. Effectiveness of an in-office arginine-calcium carbonate paste on dentine hypersensitivity in periodontitis patients: a double-blind, randomized controlled trial. J Clin Periodontol. 2015;42(1):37-45.

25. Yilmaz HG, Kurtulmus-Yilmaz S, Cengiz E. Long-term effect of diode laser irradiation compared to sodium fluoride varnish in the treatment of dentine hypersensitivity in periodontal maintenance patients: a randomized controlled clinical study. Photomed Laser Surg. 2011;29(11):721-5.

26. Huskisson EC. Measurement of pain. Lancet. 1974;2(7889):1127-31.

27. Schiff T, Dotson M, Cohen S, De Vizio W, McCool J, Volpe A. Efficacy of a dentifrice containing potassium nitrate, soluble pyrophosphate, PVM/MA copolymer, and sodium fluoride on dentinal hypersensitivity: a twelve-week clinical study. J Clin Dent. 1994;5 Spec No:87-92.

28. Douglas de Oliveira DW, Oliveira ES, Mota AF, Pereira VH, Bastos VO, Gloria JC, Goncalves PF, Flecha OD. Effectiveness of Three Desensitizing Dentifrices on Cervical Dentin Hypersensitivity: A Pilot Clinical Trial. J Int Acad Periodontol. 2016;18(2):57-65.

29. Neuhaus KW, Milleman JL, Milleman KR, Mongiello KA, Simonton TC, Clark CE, Proskin HM, Seemann R. Effectiveness of a calcium sodium phosphosilicatecontaining prophylaxis paste in reducing dentine hypersensitivity immediately and 4 weeks after a single application: a double-blind randomized controlled trial. J Clin Periodontol. 2013;40(4):349-57.

30. Orsini G, Procaccini M, Manzoli L, Giuliodori F, Lorenzini A, Putignano A. A double-blind randomized-controlled trial comparing the desensitizing efficacy of a new dentifrice containing carbonate/hydroxyapatite nanocrystals and a sodium fluoride/potassium nitrate dentifrice. J Clin Periodontol. 2010;37(6):510-7.

31. Hirsiger C, Schmidlin PR, Michaelis M, Hirsch C, Attin T, Heumann C, Domejean S, Gernhardt CR. Efficacy of $8 \%$ arginine on dentin hypersensitivity: a multicenter clinical trial in 273 patients over 24 weeks. J Dent. 2019:83:1-6.

32. Pashley DH. Dynamics of the pulpo-dentin complex. Crit Rev Oral Biol Med. $1996 ; 7(2): 104-33$.
33. Lin PY, Cheng YW, Chu CY, Chien KL, Lin CP, Tu YK. In-office treatment for dentin hypersensitivity: a systematic review and network meta-analysis. J Clin Periodontol. 2013;40(1):53-64.

34. Creeth J, Maclure R, Seong J, Gomez-Pereira P, Budhawant C, Sufi F, Holt J, Chapman N, West N. Three randomized studies of dentine hypersensitivity reduction after short-term SnF2 toothpaste use. J Clin Periodontol. 2019; 46(11):1105-15.

35. Hall C, Mason S, Cooke J. Exploratory randomised controlled clinical study to evaluate the comparative efficacy of two occluding toothpastes - a 5\% calcium sodium phosphosilicate toothpaste and an $8 \%$ arginine/calcium carbonate toothpaste - for the longer-term relief of dentine hypersensitivity. J Dent. 2017;60:36-43.

36. Torres CR, Silva TM, Fonseca BM, Sales AL, Holleben P, Di Nicolo R, Borges $A B$. The effect of three desensitizing agents on dentin hypersensitivity: a randomized, split-mouth clinical trial. Oper Dent. 2014;39(5):E186-94.

37. Holland GR, Narhi MN, Addy M, Gangarosa L, Orchardson R. Guidelines for the design and conduct of clinical trials on dentine hypersensitivity. J Clin Periodontol. 1997;24(11):808-13.

38. West NX, Seong J, Davies M. Management of dentine hypersensitivity: efficacy of professionally and self-administered agents. J Clin Periodontol. 2015;42(Suppl 16):S256-302.

39. He T, Chang J, Cheng R, Li X, Sun L, Biesbrock AR. Clinical evaluation of the fast onset and sustained sensitivity relief of a $0.454 \%$ stannous fluoride dentifrice compared to an $8.0 \%$ arginine-calcium carbonate-sodium monofluorophosphate dentifrice. Am J Dent. 2011;24(6):336-40.

40. Li Y, Lee S, Zhang YP, Delgado E, DeVizio W, Mateo LR. Comparison of clinical efficacy of three toothpastes in reducing dentin hypersensitivity. J Clin Dent. 2011;22(4):113-20.

41. Schiff T, Mateo LR, Delgado E, Cummins D, Zhang YP, DeVizio W. Clinical efficacy in reducing dentin hypersensitivity of a dentifrice containing 8.0\% arginine, calcium carbonate, and 1450 ppm fluoride compared to a dentifrice containing $8 \%$ strontium acetate and 1040 ppm fluoride under consumer usage conditions before and after switch-over. J Clin Dent. 2011; 22(4):128-38.

42. West N, Newcombe RG, Hughes N, Mason S, Maggio B, Sufi F, Claydon N. A 3-day randomised clinical study investigating the efficacy of two toothpastes, designed to occlude dentine tubules, for the treatment of dentine hypersensitivity. J Dent. 2013;41(2):187-94.

43. Ayad F, Ayad N, Zhang YP, DeVizio W, Cummins D, Mateo LR. Comparing the efficacy in reducing dentin hypersensitivity of a new toothpaste containing $8.0 \%$ arginine, calcium carbonate, and 1450 ppm fluoride to a commercial sensitive toothpaste containing $2 \%$ potassium ion: an eightweek clinical study on Canadian adults. J Clin Dent. 2009;20(1):10-6.

44. Nathoo S, Delgado E, Zhang YP, DeVizio W, Cummins D, Mateo LR. Comparing the efficacy in providing instant relief of dentin hypersensitivity of a new toothpaste containing $8.0 \%$ arginine, calcium carbonate, and 1450 ppm fluoride relative to a benchmark desensitizing toothpaste containing $2 \%$ potassium ion and $1450 \mathrm{ppm}$ fluoride, and to a control toothpaste with 1450 ppm fluoride: a three-day clinical study in New Jersey, USA. J Clin Dent. 2009;20(4):123-30.

45. Chu CH, Lo EC. Immediate post-application effect of professional prophylaxis with $8 \%$ arginine-calcium carbonate desensitizing paste on hypersensitive teeth. A practitioner-based clinical trial. Am J Dent. 2014; 27(1):7-11.

46. Elias Boneta AR, Galan Salas RM, Mateo LR, Stewart B, Mello S, Arvanitidou LS, Panagakos F, DeVizio W. Efficacy of a mouthwash containing $0.8 \%$ arginine, PVM/MA copolymer, pyrophosphates, and 0.05\% sodium fluoride compared to a commercial mouthwash containing $2.4 \%$ potassium nitrate and $0.022 \%$ sodium fluoride and a control mouthwash containing $0.05 \%$ sodium fluoride on dentine hypersensitivity: a six-week randomized clinical study. J Dent. 2013;41(Suppl 1):S34-41.

47. Wara-aswapati N, Krongnawakul D, Jiraviboon D, Adulyanon S, Karimbux N, Pitiphat $W$. The effect of a new toothpaste containing potassium nitrate and triclosan on gingival health, plaque formation and dentine hypersensitivity. J Clin Periodontol. 2005;32(1):53-8.

\section{Publisher's Note}

Springer Nature remains neutral with regard to jurisdictional claims in published maps and institutional affiliations. 